\title{
An Analysis of Complications of Neck Dissection in Head and Neck Cancers
}

\author{
Shashidhar Kallappa ${ }^{1}$, Prajwal Dange ${ }^{2}$ \\ ${ }^{1}$ Department of Surgical Oncology, Karnataka Institute of Medical Sciences, Hubli, India \\ ${ }^{2}$ Department of Otorhinolaryngology, Karnataka Institute of Medical Sciences, Hubli, India
}

Email address:

Drshashi75@rediffmail.com (S. Kallappa), Prajwal.dange@gmail.com (P. Dange)

\section{To cite this article:}

Shashidhar Kallappa, Prajwal Dange. An Analysis of Complications of Neck Dissection in Head and Neck Cancers. International Journal of Clinical Oncology and Cancer Research. Vol. 5, No. 2, 2020, pp. 24-28. doi: 10.11648/j.ijcocr.20200502.12

Received: April 12, 2020; Accepted: May 3, 2020; Published: May 19, 2020

\begin{abstract}
The neck dissection has remained a pivotal aspect of head and neck cancer management for over a century. During this time its role has expanded from a purely therapeutic option to an elective setting. Since vital anatomical structures are close, certain risks and complications are inherent to this procedure. Since neck surgery remains the most frequently performed form of therapeutic surgery in head and neck cancer irrespective of primary disease site, our objective is to report the complications in various types neck dissections and to seek improved outcome. A cross sectional retrospective study of 52 patients who underwent neck dissection from August 2015 to August 2019 was conducted to analyse intra operative and post-operative complications which aroused due to neck dissection. Indications for neck dissection depended on neck staging (N): selective neck dissection was done when evident disease was absent; Modified radical neck dissection was done if there was clinically evident neck node, preserving non-lymphatic neck structures (accessory nerve, internal jugular vein and internal jugular vein) as long as surgical completeness was not compromised. Bilateral neck dissection was indicated if contralateral disease was suspected or present. Out of 52 patients, one radical neck dissection, 14 modified radical and 37 selective neck dissection, of which 32 underwent supra omohyoid neck dissection and 5 underwent anterolateral and posterolateral neck dissection. The most frequent complication was marginal mandibular nerve injury (5.5\%), followed by accessory nerve injury (2.1\%). There was one death. A careful preoperative assessment of the patient, meticulous surgical techniques, good-quality postoperative care and appropriate rehabilitation are the cornerstones of preventing and managing complications of neck dissection.
\end{abstract}

Keywords: Neck Dissection Complications, Head and Neck Cancers, Marginal Mandibular Nerve Injury, Spinal Accessory Nerve Injury, IJV Injury

\section{Introduction}

The presence of cervical lymph node metastasis is one of the most important prognostic factors in head and neck cancer management. Neck dissection is an important surgical procedure in diagnosis (staging) and treatment of cancers of head and neck. It consists of removing lymph nodes from specific areas of the neck with or without removing the sternocleidomastoid muscle, the internal jugular vein, and the accessory nerve. Since vital anatomical structures are close, risks and complications are inherent to this procedure [1]. Postoperative complications of neck dissection have a significant impact on morbidity including fatal outcomes and health care cost because of prolonged hospitalization, revision operations and permanent disability.
Since Crile [2] introduced radical Neck Dissection at the beginning of the 20th century, a few changes have been proposed, in particular Suárez's [3] functional Neck Dissection, which aimed for a more conservative approach to preserve vital anatomical structures in the neck without compromising the completeness of lymph node removal. The transition from radical to selective Neck Dissection has resulted in lesser complications and low morbidity and has preserved compliance with the oncologic principles [4].

\section{Material and Methods}

A total of 52 patients who underwent various neck dissection procedures from October, 2015 to January, 2019, were retrospectively evaluated for complications and rates of 
complications.

Indications for neck dissection depended on neck staging $(\mathrm{N})$ : selective neck dissection was done when evident disease was absent: Modified radical neck dissection was done if there were clinically evident neck lymph nodes, preserving non-lymphatic neck structures (accessory nerve, internal jugular vein and sternocleidomastoid muscle) as long as surgical completeness was not compromised. Selective or radical bilateral neck dissection was indicated if contralateral disease was suspected.

Co-morbidities conditions such as hypertension, diabetes, relative malnutrition cardiac and respiratory disease were optimized before posting the patient for surgery to minimize risk of complications. Adequate nutritional care should be given to the patient before taking for the surgery with either a nasogastric tube or percutaneous gastrostomy. Postoperatively general systemic complications were dealt with high quality health care with the help of anesthetist and physicians.

The surgeries were performed under strict asepsis and the operative area was shaved before surgery. Selective and comprehensive neck dissection (MRND and RND) were performed using appropriate incisions and elevation of the flaps was strictly sub-platysmal. Negative suction drains were kept for all the cases and removed when the drain was $<20 \mathrm{ml}$ in volume and serosanguinous in nature. A collar dressing was done and check dressing performed after 3 days. Sutures removal was done on the 10th postoperative day.

Immediate complications occurred during the operation or immediately after and manifested within 24 hours after surgery. Intermediate and late complications are those which occurred on days after surgery before the patient is discharged and those occurring months or years after the procedure, respectively.

The following complications were investigated: intraoperative and postoperative hemorrhage, suture line dehiscence (epidermolysis; and deep dehiscence); infection/suppuration; chylous leakage; subcutaneous emphysema; pneumothorax; salivary leakage; cephalic vein stasis (manifested as edema/facial cyanosis, suffusion/conjunctival edema; proptosis; venous engorgement of retina; and seizures/cardio-respiratory depression); bronchopneumonia and lung complications; nerve injury (accessory nerves, marginal mandibular branch, vagus, hypoglossal, phrenic nerve and the cervical sympathetic trunk); uncommon complications arteriovenous fistula, inappropriate $\mathrm{ADH}$ secretion syndrome, cerebral vascular accident, air embolism and blindness) and intraoperative and post-operative mortality.

Observed wound complications were classified as early, intermediate and late, local and systemic. The list of various complications that may occur during neck dissections are given below in Table 1.

Table 1. Potential complications after neck dissection.

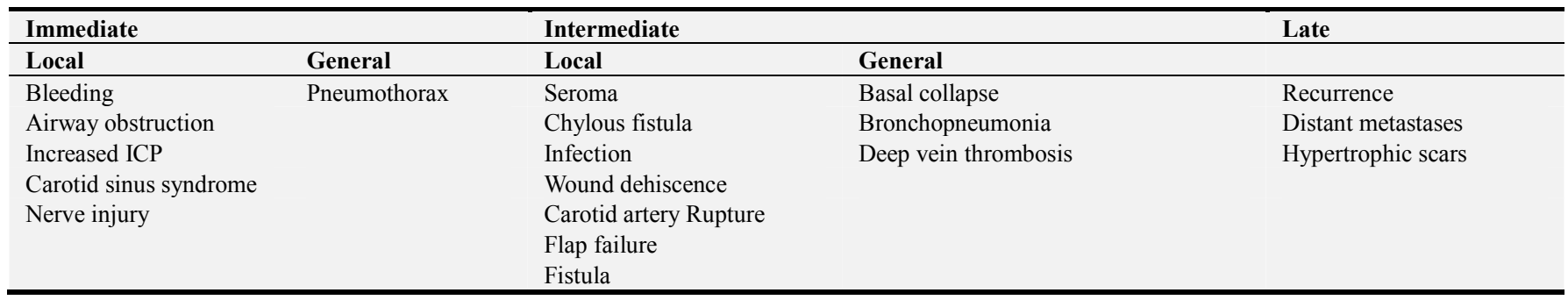

\section{Results}

The clinical charts of 52 patients were reviewed, 42 were male $(82.69 \%)$ and 10 were female $(17.30 \%)$ with a mean age of 54 years (range: $30-86$ years). The demographic and clinical characteristics are presented in Figure 1.

Most of the head and neck cancers were squamous cell carcinoma with most common pathology being well differentiated squamous cell carcinoma. Out of 52 patients, $40(76.92 \%)$ did not develop any complications, while 12 (23.07\%) experienced complication.

The major complications were marginal mandibular nerve injury which occurred in 4 patients $(7.69 \%)$, spinal accessory nerve injury which occurred in 3 patients $(5.76 \%)$ followed by hypoglossal nerve injury which occurred in 1 patient $(1.92 \%)$. Wound dehiscence occurred in 1 patient $(1.92 \%)$ and secondary suturing was done for the same. Two patients (3.84\%) had accidental injury to internal jugular vein, a small tear of $5-8 \mathrm{~mm}$ was present and it was repaired using 5.0 proline suture material. No postoperative bleeding. There was one $(1.92 \%)$ death, a case of buccal mucosa malignancy who underwent composite resection with PMMC flap reconstruction who was deceased on the second postoperative day due to aspiration pneumonia. The complication rate and types of complications are given in the Figure 2.

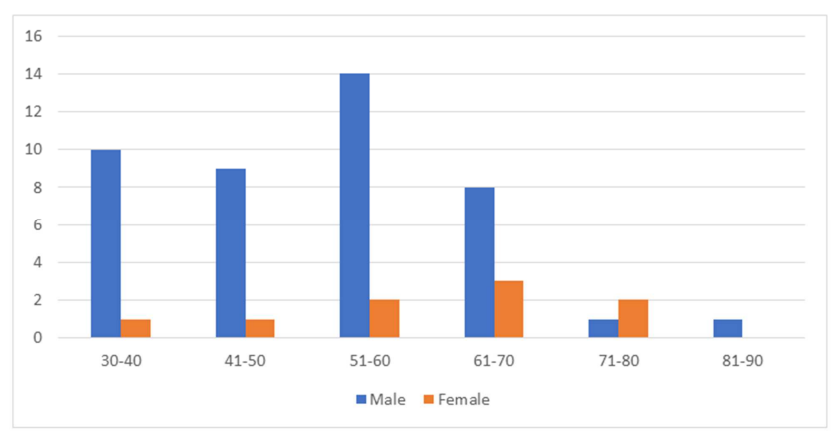

Figure 1. Age distribution in the cases of malignancy. 


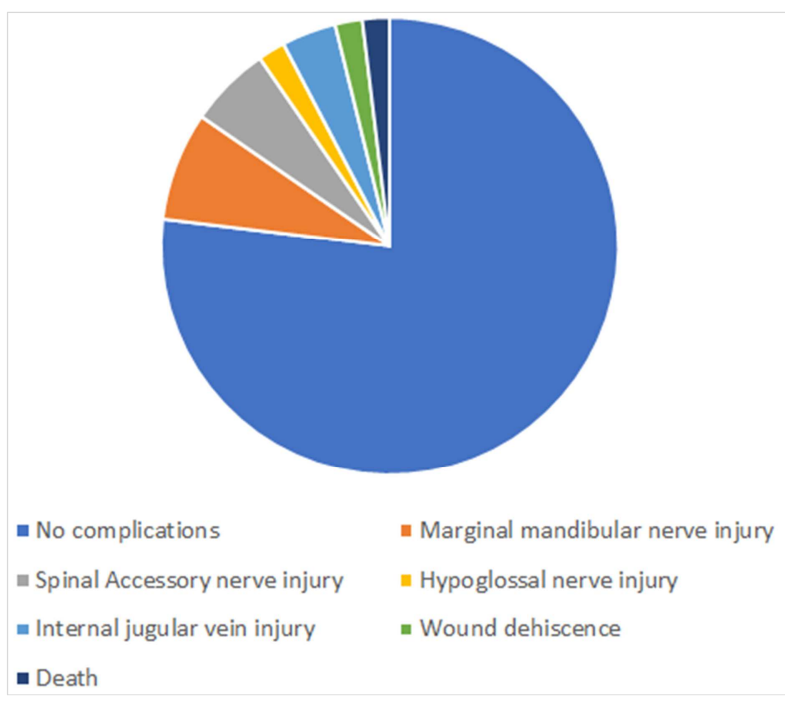

Figure 2. Complications of neck dissection.

\section{Discussion}

There are several factors which affect the prognosis in hand and neck cancers, most important among them are lymph node metastasis, level and its size. The systemic burden of the malignancy can be measured by these factors an indirect index. Therefore, elective removal of regional lymph nodes serves as a biopsy staging procedure to ascertain whether or not metastatic disease is present and to identify high risk patients who might benefit from systemic adjuvant therapy [5].

The RND for palpable or potential cervical metastasis from head and neck cancer remained the standard treatment for many decades since its original description by Crile in 1906 and subsequent popularization by Martin in 1951 [2]. The morbidity and complications associated with RND became apparent while it was a reliable method of treating patients with head and neck cancer. The change in type of operation performed to deal with metastatic disease was due to the greater understanding of patterns of metastatic disease for the various primary sites. In the study which we conducted out of 51 patients 40 patient $(76.92 \%)$ did not develop any complications which is comparable with the study conducted by Monika S et al [6] in which 66 patients $(80.48 \%)$ out of 82 patients did not develop any complications.

The infection following head and neck surgery can either local or systemic. Systemic infection is fortunately rare but may arise if local infection is inadequately treated. In the study we conducted wound dehiscence occurred in 1 patient (1.92\%) out of 52 patients compared to 3 patients (3.65\%) out of 82 patients in a study conducted by Monika $S$ et al [6]. Localized infections can occur around intravenous access sites, while prolonged urethral catheterization will predispose to urinary tract infection. Early mobilization will prevent skin breakdown and development of cutaneous infection. Local wound infection is uncommon after clean procedures when no mucosal surface is open (e.g. neck dissection). In these circumstances prophylactic antibiotics are not required and careful wound care will suffice [7]. However, in cleancontaminated operations, such as laryngectomy, prophylactic antibiotic use is indicated. Standard practice usually involves a single intraoperative dose of broad-spectrum antibiotic followed by 24-48 hours of antibiotic [7]. There is little evidence in the literature to support the use of longer courses of antibiotics [8,9]. The concurrent en-bloc resection of a primary oral or oro-pharyngeal lesion result in a throughand-through defect. This leads to increased rate of wound infection, although benefits of topical treatments with either antiseptics or antibiotics have been demonstrated [10]. The rate of infection can be minimized to an extent by meticulous suturing. The oral mucosal defects can be sutured in two layers as it increases the strength and reduces dehiscence. Intra-venous antibiotics reduces the length of peri-operative treatment by limiting the development of drug-resistant bacterial infection, e.g., methicillin-resistant Staphalococcus aureus [11]. Since there are a variety of approaches for neck dissections and it rely on the preference of surgeon. The external carotid artery and the subclavian artery are the important vascular supply of cervical skin. In our study, Selective neck dissection and comprehensive neck dissection (MRND and RND) were performed via appropriate incision. The elevation of flaps was strictly sub-platysmal in all the dissections and closure was done in two layers (platysma with subcutaneous tissue in one layer and skin in another layer) which aided in better healing of wound. A suction drain was kept in all cases during skin closure. Out of the 52 cases. Drain acts by removing the serous collection and its management has important implications in wound healing. Infection at the surgical wound site can be reduced by placing the drain separately from the incision. Drain reservoirs should be monitored frequently to ensure adequate compression, particularly in the first $24 \mathrm{~h}$ after insertion [12].

In the study which we conducted we encountered $7.69 \%$ marginal mandibular nerve injury, which occurred when the upper flap is elevated or during level 1 dissection (submental/submandibular triangles); it may cause dysfunction of the lower lip depressor muscle. This injury was encountered in $18 \%$ of patients and $23 \%$ of necks in a study of 66 patients undergoing ND, resulting in an asymmetric smile but no severe sequelae in study conducted by Batstone et al, [13] and in study conducted by Prim et al, [14] A lesion of the marginal branch of the 7th cranial nerve was observed in nine cases $(1.26 \%)$. The overall neuropraxia rate in a heterogeneous group of patients undergoing neck dissection has been described at $16 \%$ [15]. The excellent anatomical knowledge and also the awareness of various variations in course of the nerve help to prevent the injury while working in the vicinity of nerves. Intraoperative nerve monitoring is increasingly popular with surgeons in an attempt to avoid nerve injury [16]. Whenever possible the cranial nerves integrity should be maintained unless tumor resection is compromised. Marginal mandibular nerve is the most important branch of facial nerve supplying the circumoral musculature which 
should be preserved while raising the upper flap. This nerve curves below and in front of the angle of mandible [17] and its distance from the inferior border of mandible varies from $1.4 \mathrm{~cm}$ to $1.75 \mathrm{~cm}[18,19]$. Therefore, while dissection the incision should be $>1.5 \mathrm{~cm}$ below the angle and parallel to lower border of mandible and the nerve should be raised with the flap. The Marginal mandibular nerve is mostly superficial to facial artery making it an important landmark to locate the nerve. Other important points that should be taken care of are not to use bipolar diathermy on the fall and not to transfix the nerve.

Neurological complications were diagnosed on a clinical basis [20]. The findings in the 11 th nerve paralysis were the following: pain in the shoulder, limited abduction of the shoulder, and a full passive range of motion [14]. In our study $5.76 \%$ of cases had spinal accessory nerve injury which is comparable with Paralysis of the $11^{\text {th }}$ nerve occurred in 12 cases $(1.68 \%)$ in a study conducted by Prim et al, [14]. Syndrome of decreased range of abduction in the shoulder joint and pain following RND is due to sacrifice of the Spinal Accessory Nerve. Preservation of that structure during neck dissection helps in ameliorating the syndrome [21]. The development of modified procedures has helped to reduce the adverse effects of the classical operation and yet preserve its effectiveness in oncological terms. Nevertheless, if the 11th nerve is transected during the procedure, the management is twofold. Immediate intraoperative options consist of a very careful preservation of the $\mathrm{C} 2, \mathrm{C} 3$, and $\mathrm{C} 4$ branches as long as this appears to be oncologically feasible. The reason for this is that although motor contribution of the cervical branches is not consistent or significant from an anatomical standpoint [22], some improvement in the range of motion (specially exorotation and anteflexion) is observed when these branches are spared [23]. On the other hand, primary anastomosis of nerve endings or cable grafting have also been proposed [21]. This cable grafting restores some function of the trapezius muscle through reinnervation. If significantly shoulder pain and disability is apparent in the early postoperative phase then progressive resistance exercise training should be instituted in an attempt to minimize its impact and improve quality of life [24].

Bernard-Horner's syndrome and hypoglossal nerve paralysis were noted in four and three cases (0.56 and $0.42 \%$ ) respectively in study conducted by Prim et al, [14] which is comparable with our study in which $1.92 \%$ of cases who underwent neck dissection had hypoglossal nerve injury. Lesions of the hypoglossal nerve impair tongue movement with problems linked to food intake and speech articulation. Its damage is related to procedures in and around the carotid artery. In the same way as other cranial nerves, the best treatment is careful identification in order to preserve the nerve, if possible. If the hypoglossal nerve is accidentally transected or if a portion must be sacrificed secondary to tumor involvement, primary anastomosis or cable grafting seems justified. Nevertheless, the results are not predictable [25]. Thus, rehabilitation appears to be the most reasonable approach to this deficit
In our study $2(3.84 \%)$ of cases had accidental injury to internal jugular vein, a small tear of $5-8 \mathrm{~mm}$ was present and it was repaired using 5.0 proline suture material which is comparable with study conducted by Bhushan SN et al, [26] in which internal jugular vein injured in $2(2.4 \%)$ cases. To avoid troublesome bleeding from the internal jugular vein (IJV), it should be mobilized circumferentially using a spreading motion perpendicular to the vessel wall. In the event of a large accidental IJV rent, this can be repaired using a running 6-0 vascular suture. It is good practice to tie off tributaries away from the IJV wall to avoid eddy currents and subsequent thrombosis formation. Injury to vagus, hypoglossal and accessory nerves should be avoided while ligating the vessel. Troublesome bleeding can ensue if either end of the IJV retracts. Packing with hemostatic sponge may control bleeding if the superior pole retracts into the temporal bone, failing which one may need to skeletonize the jugular vein or sigmoid sinus to control the bleed. Similarly, a sternal split may rarely be needed if the lower stump retracts into the mediastinum [7].

\section{Conclusion}

The neck dissection has become the accepted part of head and neck oncology over the past century, be it performed either in isolation or as an integral element of a more major resection and reconstruction hence a careful preoperative assessment, meticulous surgical technique, high quality postoperative care and appropriate rehabilitation are the cornerstones of preventing and managing complications. Modified procedures reduces the adverse effects of the classical operation and yet preserve its effectiveness in oncological terms. Complications can still occur despite the best planning but their impact can be minimized by a vigilant and proactive emphasis in the entire peri-operative period.

\section{References}

[1] Smullen JL, Lejeune FEJ. Complications of neck dissection. J La State Med Soc. 1999 Nov; 151 (11): 544-7.

[2] Crile G. Excision of Cancer of the Head and Neck. With special reference to the plan of dissection based on 132 patients. Jama. 1906; 47: 1780-6.

[3] Suarez O. El problema de las metastasis linfáticas y alejadas del cáncer de laringe e hipofaringe. Rev Bras Otorrinolaringol. $1963 ; 23,: 83-99$.

[4] Ferlito A, Gavilàn J, Buckley JG, Shaha AR, Miodoński AJ, Rinaldo A. Functional neck dissection: Fact and fiction. Head Neck. 2001 Sep 1; 23 (9): 804-8.

[5] Paleri V WJ. in: Stell \& Maran's Textbook of Head and Neck Surgery and Oncology. 5th ed. London: Hodder Arnold. 2012. $663 \mathrm{p}$.

[6] Malgonde M, Kumar M. Complications after neck dissection. Med J Dr DY Patil Univ. 2015; 8 (4): 458. 
[7] Arnold H. Complications and their managment. In: Stell \& Maran's Textbook of Head and Neck Surgery and Oncology, fifth edition. 2012. p. 195-213.

[8] Coskun H, Erisen L BO. Factors affecting wound infection rates in head and neck surgery. Otolaryngol Neck Surg. 2000; 123: $328-33$.

[9] Scottish Intercollegiate Guidelines Network. Antibiotic prophylaxis in surgery, Guideline number 104,. Available from: www.sign.ac.uk/pdf/sign104.pdf.

[10] Grandis JR, Vickers RM, Rihs JD, Yu VL, Wagner RL, Kachman KK, et al. The efficacy of topical antibiotic prophylaxis for contaminated head and neck surgery. Laryngoscope. 1994; 104 (6): 719-24.

[11] Walker FDL, Cooke LD. Antimicrobial prophylaxis in otorhinolaryngology/head and neck surgery. Clin Otolaryngol. 2007; 32 (5): 405.

[12] Williams J, Toews D, Prince M. Survey of the use of suction drains in head and neck surgery and analysis of their biomechanical properties. J Otolaryngol. 2003 Feb; 32 (1): $16-22$.

[13] Martin D. Batstone BS, Derek Lowe SNR. Marginal mandibular nerve injury during neck dissection and its impact on patient perception of appearance. Head Neck. 2009; 31: 673-678.

[14] Prim MP, De Diego JI, Verdaguer JM, Sastre N, Rabanal I. Neurological complications following functional neck dissection. Eur Arch Oto-Rhino-Laryngology. 2006; 263 (5): 473-6.

[15] Nason RW, Binahmed A, Torchia MG TJ. Clinical observations of the anatomy and function of the marginal mandibular nerve. Surg, Int J Oral Maxillofac. 2007; 36: 712715 .

[16] Edwards BM, Kileny PR. Intraoperative neurophysiologic monitoring: indications and techniques for common procedures in otolaryngology-head and neck surgery. Otolaryngol Clin North Am. 2005 Aug; 38 (4): 631-42, viii.
[17] Charles R RS. Rob Charles Operative Surgery. London: Butterworths; 1983; 524-5.

[18] Rödel R LJ. Peripheral branches of the facial nerve in the cheek and chin area. Anatomy and clinical consequences. HNO. 1996; 44: 572-6.

[19] Converse JM. Head and neck surgery. In: Plastic and Reconstructive Surgery 2nd ed, Vol 5 Philadelphia, Landon, Toronto: WB Saunders and Company. 1979. p. 112.

[20] Shockley WW, McQueen CT PG. Complications of neck surgery. In: Weissler MC, Pillsbury HC (eds) Complications of head and neck surgery Thieme, New York,. 1995. p. 122158.

[21] Short SO, Kaplan JN, Laramore GE CC. Shoulder pain and function after neck dissection with or without preservation of the spinal accessory nerve. Am J Surg. 1984; 148: 478-82.

[22] Nori S, Soo KC, Green RF, Strong EW MS. Utilization of intraoperative electroneurography to understand the innervation of the trapezius muscle. Muscle Nerve. 1997; 20: 279-285.

[23] El Ghani F, Van Den Brekel MWM, De Goede CJT, Kuik J, Leemans CR, Smeele LE. Shoulder function and patient wellbeing after various types of neck dissections. Clin Otolaryngol Allied Sci. 2002; 27 (5): 403-8.

[24] McNeely ML, Parliament MB, Seikaly H, Jha N, Magee DJ, Haykowsky MJ et al. Effect of exercise on upper extremity pain and dysfunction in head and neck cancer survivors: A randomized controlled trial. Cancer. 2008; 113: 214-22.

[25] Weisberger EC, Lingeman RE. Cable grafting of the spinal accessory nerve for rehabilitation of shoulder function after radical neck dissection. Laryngoscope. 1987 Aug; 97 (8 Pt 1): 915-8.

[26] Suhas N. Bhushan AHN. Management of neck dissection complications in head and neck cancers Suhas N. Bhushan, Arun H. N. Int Surg J. 2019; Mar; 6 (3): 664-8. 